\title{
De Ia política a la acción: Estado y avances de la implementación de la política pública social para el envejecimiento y la vejez (PPSEV) en Bogotá*
}

\section{From Policy to Action: State and Progress of the Implementation of the Social Public Policy for Aging and OId Age (Ppsev) in Bogota}

\author{
Ángela María Jaramillo* \\ Ángela Lucía Forero**
}

Recibido: 21/09/2015

Aprobado: 4/10/2015

Disponible en línea: 30/11/2015

\begin{abstract}
Resumen
Uno de los principales desafíos que enfrentan las políticas públicas en general y la Política Pública Social de Envejecimiento y Vejez (PPSEV) de Bogotá en particular, es la armonización entre las acciones ya desarrolladas para atender a la población mayor de la ciudad y la creación de ambientes sociales en los que las personas tengan las condiciones necesarias y suficientes para envejecer con dignidad y equidad.
\end{abstract}

\begin{abstract}
One of the main challenges facing the Politics of Aging (PPSEV) in Bogotá, is the alignment between the actions that have been developed to attend the old population of the city, and the creation of social environments where people have the necessary conditions to age with dignity and fairness. This document describes the progress and challenges in the process of implementing the PPSEV, from the everyday experiences of
\end{abstract}

doi:10.11144/Javeriana.papo20-2.paea

* Este artículo hace parte de la investigación: "De los hechos a la acción: focalización e impacto de la Política Distrital de Envejecimiento y Vejez". Línea de base construida con la Encuesta de Salud, Bienestar y Envejecimiento. (SABE Bogotá, 2012). Desarrollado por el Instituto de Envejecimiento y el Departamento de Sociología de la Pontificia Universidad Javeriana. Las fuentes utilizadas para la elaboración de este artículo son documentos y entrevistas que se encuentran disponibles en el Instituto de Envejecimiento.

* Socióloga de la Universidad Nacional de Colombia. Magíster en Estudios de Población, y Doctoranda en Estudios Sociales de la Universidad Externado de Colombia. Docente investigadora de la Pontificia Universidad Javeriana, Bogotá. Correo electrónico: jaramillo-angela@javeriana.edu.co ** Socióloga y Economista de la Pontificia Universidad Javeriana. Correo electrónico: forero-angela@ javeriana.edu.co 
En el presente documento se describen los avances y las dificultades que enfrenta el distrito en el proceso de implementación de la PPSEV a partir de las experiencias cotidianas de los coordinadores y profesionales encargados de desarrollar el proyecto 742, el cual implementa esta política pública. La percepción, funciones y actividades de los gestores son aspectos relevantes para reflexionar sobre la conexión entre los elementos técnicos (planes de acción, metas e indicadores) y el marco de acción formulado. La coherencia entre los referentes conceptuales de la política y su ejecución facilita el alcance de las metas y resultados de la PPSEV y en consecuencia, la creación de contextos adecuados para una sociedad de todas las edades.

\section{Palabras clave}

envejecimiento; vejez; implementación; política social

\section{Cómo citar este artículo:}

Jaramillo, A. M., \& Forero, Á. M. (2015). De la política a la acción: Estado y avances de la implementación de la política pública social para el envejecimiento y la vejez (PPSEV) en Bogotá. Papel Político, 2O(2), 295-322. http://dx.doi.org/10.11144/papo20-2.paea coordinators and professionals responsible for developing project 742 , which operationalizes the described policy. The perception, functions and activities of managers are relevant to reflect the connection between the technical elements (action plans, targets and indicators) and the formulated situation. The consistency between the conceptual aspects and performance of the policy facilitates the achievement of goals and outcomes of PPSEV and consequently creates appropriate contexts for a society for all ages.

\section{Keywords}

aging; old age; performance; social policy 


\section{Introducción}

En este documento se presenta el marco conceptual y normativo que orienta la política distrital de envejecimiento y vejez, en contraste con los avances de su implementación. Esto con el fin de conocer los adelantos y las dificultades que enfrentan las personas encargadas de desarrollar el proyecto $742^{1}$, así como las condiciones sociales que facilitan o limitan su adecuado avance. Con esta mirada se busca reconocer la complejidad del proceso de implementación de la política para contribuir en la búsqueda por armonizar la política y sus circunstancias sociales, así como en el esfuerzo por superar la tradición asistencialista.

La importancia de observar el proceso de la implementación de la política se relaciona con la continuidad existente entre las fases de elaboración y acción. Lo anterior implica la participación de los actores que diseñaron la política y de los que depende la acción. Los procesos de concertación que se alcanzan tanto en las relaciones intra-organizacionales como inter-organizacionales de los distintos niveles de gobierno, al igual que con las organizaciones privadas y sociales, permiten una adecuada implementación. Por eso es relevante identificar las tensiones que se van presentando a medida que se desarrolla la política. En este estudio se registran las experiencias de varios de los profesionales encargados de la acción, como una primera aproximación descriptiva del proceso de implementación.

\section{La política distrital de envejecimiento y vejez}

La política pública social para el envejecimiento y la vejez del Distrito Capital 2010-2025 (PPSEV) nace dentro del programa de gobierno del Alcalde Samuel Moreno, "Bogotá positiva" como una medida para garantizar, restablecer y ejercer los derechos individuales y colectivos de los habitantes de Bogotá. Se diseñó para garantizar independientemente de las circunstancias, los derechos de las personas mayores. Con este fin, se designó como operadores principales a la Secretaría de Integración Social junto con la Secretaría Distrital de Salud (Acaldía Mayor de Bogotá, 2010).

Esta política recoge los esfuerzos que el Distrito venía forjando desde 1999 cuando el Departamento Administrativo de Bienestar Social capacitó a las personas mayores en Derechos Humanos, fomentando ejercicios ciudadanos de participación desde el nivel local, como los Consejos Locales de Personas Mayores y el Consejo Distrital de Persona Mayor. Con este último se incentiva la creación en el año 2000 de la Asociación Nacional para la Defensa de los Programas y Derechos de la Tercera Edad (ANDATER), institución que buscaba mejorar sus condiciones de vida.

\footnotetext{
${ }^{1}$ Dado el carácter de largo alcance propuesto por la política, esta se articula al Plan de Desarrollo Distrital (PDD) del Alcalde Gustavo Petro "Bogotá Humana" (2012-2016) por medio del proyecto 742 que actualmente operacionaliza la atención integral al adulto mayor en el Distrito.
} 
Adicionalmente en el 2003 el Consejo Distrital de Política Social creó un primer documento de pautas de política social: los lineamientos generales de política social para Bogotá 2004-2014 que conceptualizaban la política pública como un grupo de valores, principios, propósitos y lineamientos que permitirían direccionar las acciones de la ciudad. Desde ese entonces la formulación de políticas en el Distrito ha tratado de articularse con las ya existentes, complementándose y comunicándose mutuamente para responder de manera más adecuada a las realidades sociales de la ciudad.

Durante el 2007 se dio una reforma administrativa en el Distrito y se creó la Secretaría de Integración Social, ente encargado de la formulación y ejecución de políticas sociales en Bogotá. Uno de los temas en los que se centra la institución es la vejez y por ende, vuelve primordial la formulación de una política pública de envejecimiento y vejez que trascienda las políticas asistencialistas hasta entonces vigentes y que otorgue autonomía al adulto mayor como sujeto político. El año anterior el Consejo de Bogotá había expedido el Acuerdo 254 que establecía los lineamientos de la política pública de envejecimiento y las personas mayores en el Distrito Capital.

La participación de la opinión pública en la construcción de la PPSEV se dio por medio de la consulta social. Esta recogió las diferentes opiniones de la sociedad civil, organizaciones gubernamentales y no gubernamentales, respecto a algunas de las orientaciones dadas por el Plan de Acción Internacional de Madrid sobre Envejecimiento (Naciones Unidas, 2003): 1) Las personas de edad y el desarrollo; 2) El fomento de la salud y el bienestar en la vejez; y 3) La creación de un entorno propicio y favorable (Acaldía Mayor de Bogotá, 2010, p. 17).

Con este propósito en mente y desde una visión participativa de la sociedad, la política se plantea el objetivo de:

Garantizar la promoción, protección, restablecimiento y ejercicio pleno de los derechos humanos de las personas mayores sin distingo alguno, que permita el desarrollo humano, social, económico, político, cultural y recreativo, promoviendo el envejecimiento activo para que las personas mayores de hoy y del futuro en el Distrito Capital vivan una vejez con dignidad, a partir de la responsabilidad que le compete al Estado en su conjunto y de acuerdo con los lineamientos nacionales e internacionales. (Acaldía Mayor de Bogotá, 2010, p. 69)

Muestra unas intenciones específicas que destacan el respeto por la autonomía, la creación de entornos ambientales y sociales favorables, la coordinación de las redes de protección y la transformación de los imaginarios sociales.

La materialización de la política parte de estas aspiraciones con un enfoque de derechos basado en un valor principal tanto para la Declaración de Derechos Humanos como para la Constitución de 1991 -y el Estado-y la dignidad humana. Es así que se crean las 
dimensiones, ejes y líneas a través de los cuales se desarrolla la implementación de la política. Del valor surgen los principios de igualdad, equidad y diversidad que orientan las cuatro dimensiones de la política, de las que se desprenden los ejes a través de los que se enmarcan las acciones y estrategias que toma para implementarse en el territorio.

\section{Cuadro 1. Estructura de la PPSEV}

\begin{tabular}{|c|c|c|c|}
\hline $\begin{array}{c}\text { VALOR Y } \\
\text { PRINCIPIOS }\end{array}$ & DIMENSIONES & EJES & LÍNEAS \\
\hline & $\begin{array}{l}\text { 1.Vivir como se quiere en la vejez } \\
\text { Reconoce y potencia, la autonomía y } \\
\text { la libertad individual en la construcción } \\
\text { de proyectos de vida de las personas } \\
\text { mayores; implica la capacidad de elegir y }\end{array}$ & $\begin{array}{l}\text { Decidiendo y } \\
\text { viviendo a mi manera }\end{array}$ & $\begin{array}{l}\text {-Libre desarrollo de la } \\
\text { personalidad } \\
\text {-Libertad de } \\
\text { conciencia, } \\
\text { religiosa y de culto } \\
\end{array}$ \\
\hline & $\begin{array}{l}\text { tomar decisiones frente a las situaciones } \\
\text { que se presentan cotidianamente, marca } \\
\text { el curso de las estrategias y acciones } \\
\text { dirigidas a la garantía de la autonomía y la } \\
\text { participación de las personas mayores. }\end{array}$ & $\begin{array}{l}\text { Construyendo el bien } \\
\text { común }\end{array}$ & $\begin{array}{l}\text {-Información, } \\
\text { comunicación y } \\
\text { conocimiento } \\
\text {-Participación }\end{array}$ \\
\hline & $\begin{array}{l}\text { 2.Vivir bien en la vejez } \\
\text { Es la dimensión patrimonial de la dignidad } \\
\text { humana. Hace referencia a las condiciones } \\
\text { materiales de existencia de las personas } \\
\text { mayores; permite gozar de un real y }\end{array}$ & $\begin{array}{l}\text { Techo, comida y } \\
\text { sustento }\end{array}$ & $\begin{array}{l}\text {-Seguridad económica } \\
\text {-Trabajo } \\
\text {-Vivienda } \\
\text {-Alimentación y } \\
\text { nutrición }\end{array}$ \\
\hline & $\begin{array}{l}\text { efectivo acceso, calidad, permanencia y } \\
\text { disfrute de bienes y servicios que permitan } \\
\text { vivir bien y en sociedad teniendo en } \\
\text { cuenta las condiciones y diversidades }\end{array}$ & $\begin{array}{l}\text { Seres saludables y } \\
\text { activos }\end{array}$ & $\begin{array}{l}\text {-Salud } \\
\text {-Educación } \\
\text {-Cultura } \\
\text {-Recreación y deporte }\end{array}$ \\
\hline & & $\begin{array}{l}\text { Entorno sano y } \\
\text { favorable }\end{array}$ & $\begin{array}{l}\text {-Ambiente sano } \\
\text {-Movilidad }\end{array}$ \\
\hline & $\begin{array}{l}\text { 3.Vivir sin humillaciones en la vejez } \\
\text { Es la expresión de la intangibilidad de } \\
\text { ciertos bienes no patrimoniales, como } \\
\text { la integridad física, psicológica y moral }\end{array}$ & $\begin{array}{l}\text { Respetándonos y } \\
\text { queriéndonos }\end{array}$ & $\begin{array}{l}\text {-Seguridad e } \\
\text { integridad }\end{array}$ \\
\hline & $\begin{array}{l}\text { reconocimiento y respeto de la familia, } \\
\text { la sociedad y el Estado, del valor y lugar } \\
\text { que deben tener las personas mayores, } \\
\text { restituyendo y garantizando su integridad } \\
\text { cuando esta ha sido vulnerada. }\end{array}$ & $\begin{array}{l}\text { Cuidándome y } \\
\text { cuidándonos }\end{array}$ & $\begin{array}{l}\text {-Acceso a la justicia } \\
\text { - Protección y cuidado }\end{array}$ \\
\hline \multirow{3}{*}{ 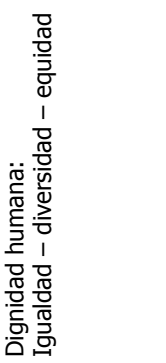 } & \multirow{3}{*}{$\begin{array}{l}\text { 4.Envejecer juntos y juntas } \\
\text { Reconoce y visibiliza el envejecimiento } \\
\text { como un proceso natural, diverso y } \\
\text { continuo, relacionando y poniendo a } \\
\text { dialogar la vejez con los demás momentos } \\
\text { del ciclo vital con el fin de transformar } \\
\text { los imaginarios y prácticas adversas y } \\
\text { discriminatorias, implantando la cultura } \\
\text { del envejecimiento activo que fortalezca } \\
\text { valores, saberes y prácticas de las } \\
\text { personas mayores de hoy y del futuro. }\end{array}$} & $\begin{array}{l}\text { Escuchando nuestros } \\
\text { saberes }\end{array}$ & $\begin{array}{l}\text {-Responsabilidad } \\
\text { intergeneracional } \\
\text {-Vínculos y relaciones } \\
\text { familiares }\end{array}$ \\
\hline & & $\begin{array}{l}\text { Aprendiendo a } \\
\text { envejecer }\end{array}$ & $\begin{array}{l}\text {-Plan de vida } \\
\text {-Gestión a la } \\
\text { investigación }\end{array}$ \\
\hline & & $\begin{array}{l}\text { Cambiando para } \\
\text { mejorar }\end{array}$ & $\begin{array}{l}\text {-Imágenes del } \\
\text { envejecimiento } \\
\text {-Formación a } \\
\text { cuidadores y } \\
\text { cuidadoras }\end{array}$ \\
\hline
\end{tabular}

Fuente: Alcaldía Mayor de Bogotá (2010)

Una segunda etapa de la política se encarga de exponer las herramientas que serán utilizadas para dar seguimiento y evaluación de la misma (páginas 92 a 99 de la PPSEV 2010-2025). Entre ellas se encuentra una batería de indicadores específicos para cada dimensión. Estos tienen la intensión de evaluar la evolución de la política a través del 
tiempo. Su periodicidad es anual y para ser elaborados, requieren de la coordinación de entidades como la Secretaría Distrital de Integración, el DANE, la Mesa Distrital de Envejecimiento, la Secretaría Distrital de Salud, la Secretaría Distrital de Ambiente, la Secretaría Distrital de Gobierno, entre otros.

\section{Cuadro 2. Ejemplos}

\begin{tabular}{|l|l|l|l|l|}
\hline DIMENSIÓN & \multicolumn{1}{|c|}{$\begin{array}{c}\text { NOMBRE DEL } \\
\text { INDICADOR }\end{array}$} & $\begin{array}{c}\text { FUENTE DE } \\
\text { INFORMACIÓN }\end{array}$ & PERIODICIDAD & \multicolumn{1}{c|}{$\begin{array}{c}\text { SECTORES } \\
\text { RESPONSABLES }\end{array}$} \\
\hline $\begin{array}{l}\text { Dimensión 1. } \\
\text { Vivir como se } \\
\text { quiere en la } \\
\text { vejez }\end{array}$ & $\begin{array}{l}\text { Hogares con personas } \\
\text { mayores, con } \\
\text { acceso a redes de } \\
\text { información y nuevas } \\
\text { tecnologías. }\end{array}$ & $\begin{array}{l}\text { Secretaría Distrital } \\
\text { de Planeación, } \\
\text { Consejo Distrital de } \\
\text { Políticas Sociales e } \\
\text { IDPAC. }\end{array}$ & Anual & $\begin{array}{l}\text { Secretaría Distrital de } \\
\text { Integración Social y } \\
\text { Alcaldías locales }\end{array}$ \\
\hline $\begin{array}{l}\text { Dimensión 4. } \\
\text { Envejecer } \\
\text { juntos y juntas }\end{array}$ & $\begin{array}{l}\text { Porcentaje de colegios } \\
\text { y universidades que } \\
\text { incluyeron la temática } \\
\text { de envejecimiento y } \\
\text { vejez dentro de sus } \\
\text { proyectos educativos. }\end{array}$ & $\begin{array}{l}\text { DANE, Colciencias, } \\
\text { todos los sectores. }\end{array}$ & Anual & $\begin{array}{l}\text { Mesa Distrital de } \\
\text { Envejecimiento y } \\
\text { Vejez }\end{array}$ \\
\hline
\end{tabular}

Fuente: Alcaldía Mayor de Bogotá (2010, pp. 94-99)

Como se verá más adelante en la segunda sección de este documento, el monitoreo y evaluación ha tenido una serie de transformaciones en cuanto a su medición debido a las restricciones y posibilidades reales a la hora de implementar la política en los diferentes territorios.

Como últimas orientaciones para la implementación se destaca la importancia de un trabajo articulado entre la Secretaría de Integración Social, la Secretaría de Salud y los demás sectores de la administración, sociedad civil y entidades de orden nacional e internacional para dar cumplimiento y continuidad a la política. Por el Decreto 460 de 2008 se fija el Comité operativo como instancia de implementación. Además se dispone que la financiación sea entregada por las entidades responsables según presupuesto anual; el seguimiento y evaluación estarán orientados por un plan de acción transectorial, según lo disponga la Secretaría de Planeación. La verificación del cumplimiento de la política se hará por medio de un informe de avance presentado al Consejo de Bogotá cada seis meses, mostrando la evolución de las actuaciones.

La importancia de la creación transectorial del plan de acción radica en el deseo de lograr una gestión social integral en el Distrito a la hora de implementar la política pública social. De esta manera la creación de acuerdos y agendas de forma coordinada muestra un diálogo entre los diferentes actores sociales, lo cual resulta fundamental para la implementación de la PPSEV en el territorio.

Una de las estrategias de la política para llevar a cabo su implementación ha sido la territorialización llevada a cabo a través de los planes de acción de cada localidad, donde se procura llegar a la población más vulnerable, o a aquellos adultos mayores interesados 
en el componente de formación que brinda la misma en todo el territorio. Por esta razón el trabajo está descentralizado y guiado desde cada subdirección de integración social local. Para este fin, la gestión transectorial entre las diferentes instituciones gubernamentales y no gubernamentales es esencial puesto que brinda un mayor campo de acción que permite atender de forma más adecuada a la población. En este ámbito, tanto el sector público y privado como la sociedad civil, son los responsables de llevar la política a un feliz término.

\section{Fuentes y metodología}

Con el propósito de tener una mirada general del desarrollo de la implementación de la política en el Distrito así como de sus diferencias locales, se definieron dos perfiles de actores encargados de la acción de la PPSEV. De una parte los coordinadores o directivos y de otra los gestores. Esto con el fin de recoger percepciones variadas sobre el proceso, que van desde las funciones de orientación y decisión, hasta las operativas de campo. Adicionalmente, se reconocieron los dos territorios que en la ciudad presentan las mayores diferencias en sus avances de implementación. La disparidad en los perfiles y lugares permite recopilar información diversificada que reduce los sesgos de las experiencias particulares y nos acerca a las tensiones y contradicciones del desarrollo de la política. Se aplicaron siete entrevistas semi-estructuradas, su número se definió por la saturación de la información. El estudio de la información se hizo mediante análisis de discurso, con base hipotético-deductiva, en la que se definen previamente las categorías del estudio a partir del objetivo general del proyecto de investigación "Conocer el estado y avance del proceso de implementación de la PPSEV".

Las entrevistas se estructuraron considerando tres categorías de análisis: 1. Experiencia de los actores: se refiere a las funciones y actividades cotidianas de los coordinadores y profesionales que trabajan en el proyecto 742 que corresponde a la PPSEV, así como su percepción general del proceso de implementación de la política, sus avances y limitaciones; 2. Implementación de la PPSEV : corresponde a la definición y desarrollo de las acciones que buscan alcanzar los objetivos de la PPSEV. Es el proceso que involucra la articulación entre los referentes conceptuales de la política y su ejecución, así como el alcance de metas y resultados; y 3. Correspondencia entre los aspectos técnicos y el marco de acción de la política: se refiere a los resultados (planes de acción, metas, indicadores) de las acciones emprendidas para el alcance de los objetivos de la PPSEV.

\section{Resultados}

\section{Implementación de la PPSEV}

Aun con las similitudes vistas entre la política de vejez y el plan de desarrollo, se hace necesario armonizar todas las políticas públicas vigentes con la línea de acción que lleva el Alcalde Gustavo Petro, con el fin de no duplicar esfuerzos e integrar las políticas a la 
línea de acción trazada por el PDD. En la medida que se ha dado la implementación de las diferentes políticas, se vio la necesidad de hacer ajustes en lo respectivo a monitoreo, seguimiento y evaluación. Hubo necesidad de reelaborar los indicadores planteados inicialmente para la PPSEV en la práctica, en la actualidad incluso, estos se encuentran en construcción. Las condiciones de los diferentes territorios que componen la ciudad obligan a pensar en la creación de nuevas herramientas para lograr una medición del impacto de las actividades que se están realizando en el marco de la política y en la creación de planes de acción local que sirvan para dar cumplimiento a los objetivos trazados en el plan de acción de 15 años.

Con este fin se creó el Decreto 171 de 2013 encargado de la estandarización de las definiciones encontradas en las políticas e incluso el mismo Plan de Desarrollo, para brindar coherencia a la hora de tomar medidas. La unificación y ajuste de los planes de acción a corto plazo -que son los instrumentos mediante los cuales las Alcaldías locales proceden a la implementación de las políticas públicas sociales y responden a los compromisos entablados con la comunidad- reciben ajustes de acuerdo a la respuesta de la población y las contingencias de cada territorio. La construcción de estos se hace de manera transversal con las instituciones que tienen alguna relación con la política en cuestión. En el segundo semestre del 2013 las Subsecretarías de Integración social construyeron sus informes de seguimiento, para dar cuenta de las actividades realizadas en el marco de la política, entre ellas los talleres de formación en desarrollo humano, línea dada desde la administración distrital.

Por medio del Proyecto 742 se ha procedido en esta administración con la implementación de la PPSEV. El objetivo del proyecto es contribuir a la reducción de la discriminación por edad y la segregación socioeconómica de las personas mayores en la ciudad por medio de la implementación de estrategias de gestión transectorial, atención integral, desarrollo de capacidades y potencialidades, transformación de imaginarios y prácticas adversas sobre el envejecimiento, así como la ampliación y fortalecimiento de la participación con incidencia de esta población. (Subdirección para la Vejez, 2012, p. 16).

La ejecución de este objetivo se realiza a través de cuatro servicios: 1. Apoyo económico, 2. Centro día, 3. Centro noche, y 4. Centro de protección, que se encuentran articulados por un enfoque de desarrollo humano que busca ir más allá de la asistencia que brindan los servicios y contribuir en el desarrollo y fortalecimiento de las capacidades y potencialidades relacionadas con la participación, incidencia, cuidado, redes sociales y familiares de la personas mayores (Secretaría Distrital de Integración Social, 2014).

Según las entrevistas realizadas a coordinadores y gestores del proyecto en distintas localidades de la ciudad, se encontró que el proceso de implementación de la política se desarrolla en continuidad con los servicios prestados desde las administraciones 
anteriores. El acceso a estos servicios tiene unos protocolos que buscan priorizar las solicitudes según el número de cupos disponibles.

Para hacer la solicitud del servicio las personas vienen, traen el recibo de la luz donde viven. El requisito fundamental es que vivan dentro de la localidad, nadie puede solicitar un subsidio si no vive aquí, trae la fotocopia de la cédula y entonces nosotros los profesionales en territorio, tenemos unos formatos establecidos para eso, los llenamos y la persona se va. Luego viene una visita domiciliaria, pero en este momento solo hacemos un concepto, en donde el profesional dice, la persona en principio amerita o no amerita el servicio, con base en la información que da. Cuando la persona no amerita, entra a una lista de espera y se queda allí hasta que haya cupo. Eso puede ser mucho tiempo. Cuando hay cupo, nosotros empezamos a sacar esas fichas, que son fichas físicas, y a darle prioridad por orden de solicitud de servicios. En ese momento se hace la visita domiciliaria, se valida la información, revisando si toda esa información inicial es cierta. Ya cuando la persona sí amerita, se le otorga el ingreso. Otras visitas son las de seguimiento de permanencia, ver si la persona puede continuar con el subsidio o si ya superó esas condiciones para poderla retirar. Hay otras visitas que son de ingreso, si cumplen con los criterios que están establecidos. El seguimiento implica revisar que la persona esté viva. (Reconstrucción a partir de algunas de las entrevistas realizadas en las diferentes localidades en 2013 y 2014)

El servicio más antiguo y reconocido a nivel distrital es el apoyo económico. Este es uno de los aspectos de mayor controversia ya que es un monto que responde a una asistencia material y no al reconocimiento de la seguridad económica como un derecho en la vejez.

El subsidio económico cuenta con diferentes modalidades, donde el responsable de generar el recurso no es únicamente SDIS sino también la Alcaldía e incluso Presidencia. Esta ampliación y diversificación hace parte de los avances que registra el proyecto en su intento por acercarse a los objetivos de la política.

El subsidio A es el primer subsidio que tuvo la Secretaría Distrital de Integración. Se dio hace muchos años por un monto de $\$ 165.000$ pesos mensuales. Hace como diez años no se volvió a dar. Las personas que aún lo tienen si llegan a morir, ya no se vuelve a dar.

Está el subsidio B, que es el subsidio que tiene la Secretaría de Integración actualmente. Se les da a las personas $\$ 120.000$ pesos de nuestros fondos, por medio de una tarjeta.

Está el subsidio C, que viene de fondos locales. Anteriormente las personas venían recibiendo por parte de la Alcaldía una cosa que se llamaba bono; la persona lo recibía y ellos iban y reclamaban un mercado. Entones para no tener esa contratación con un supermercado, todas las alcaldías locales de la ciudad se unieron con la Secretaría de Integración 
Social, hicieron un convenio para entregar ese dinero y nosotros lo distribuimos; pero el seguimiento lo siguen haciendo las alcaldías. Como es un presupuesto anual que dan las alcaldías locales, a veces las personas se quedan meses sin el servicio.

El subsidio D es un subsidio de la Nación. Eso lo maneja Prosperidad Social de Presidencia de la República desde el programa "Colombia Mayor". Se encuentra en toda la nación, es entrega de dinero en efectivo por un monto de $\$ 150.000$ pesos, cada dos meses en todo el territorio nacional y tiene unas características que hace que a ese subsidio accedan las personas más vulnerables. Para acceder, la persona tiene que tener Sisben inferior a 39 puntos. Esto quiere decir que si la persona no tiene el último Sisben, no podemos hacer nada para priorizar. Tener un Sisben de 39 puntos significa que no tienen electrodomésticos, que comparten cocina y baño, que su situación habitacional no es buena. Si son las personas más vulnerables, se les está dando menos plata. Porque eran $\$ 150.000$ pesos cada dos meses. Entonces lo que el Distrito hizo fue colocar una inyección de dinero para que quedara igualado a los otros dos subsidios. Entonces a ellos se les dan $\$ 240.000$ pesos cada dos meses.

Sin embargo, esos subsidios no son vitalicios, eso depende de las condiciones de la persona. Por eso es tan importante el seguimiento. O sea, nosotros entregamos subsidios y no soltamos a la persona ahí. Estamos permanentemente mirando cuál es la situación de ese adulto mayor y si sigue ameritando la entrega de esos recursos. (Reconstrucción a partir de algunas de las entrevistas realizadas en las diferentes localidades en 2013 y 2014)

En el caso de la población rural, se trata de dar una atención más prioritaria dentro del mismo territorio evitando el desplazamiento de las personas mayores, tanto por su comodidad como para evitar la pérdida de su identidad y la connotación de la vida en el campo. Sin embargo, afrontan algunas dificultades a la hora de acceder a servicios diferentes al apoyo económico a causa de la misma ubicación y la conformación de la zona urbana.

Las personas de la zona rural reciben el servicio de apoyo económico, que es el que tenemos actualmente. También se les ofrecen los otros servicios con los que contamos, pero la diferencia es que si ellos necesitan un servicio de esos, les tocaría venirse acá hasta la zona urbana, porque hasta ahora el proyecto apenas se está consolidando. En estos momentos se está pensando en montar un Centro día en Sumapaz. Entonces digamos que por ahora, lo más fuerte es servicio con apoyo económico. (Reconstrucción a partir de entrevista realizada en el marco de la investigación en 2013)

El tipo de subsidio y el monto otorgado dependen de cada localidad y de los lineamientos dados por la subdirección de vejez y los líderes del proyecto 742 en cada territorio. Aun así el monto y el mecanismo de entrega son iguales en toda la ciudad. 


\section{Criterios de priorización para obtener los subsidios}

Dado que los cupos están limitados por el presupuesto de las entidades públicas ya mencionadas y con el objetivo de lograr una distribución eficiente de los subsidios, SDIS ha creado una serie de criterios que permiten escoger a las personas con mayores grados de vulnerabilidad para que sean partícipes de alguno de los servicios que se prestan (ver resolución 764 de 2013). A través del conocimiento de los funcionarios, se muestran cuáles son dichos criterios.

Las mujeres deben tener 52 años o más. Los hombres desde 57 años en adelante, se están priorizando personas que tengan más de 70 años. No deben tener más de un bien inmueble. Se prioriza a las personas que tengan Sisbén y no tengan régimen contributivo, pero en ciertos casos hay personas que son beneficiarias de los hijos que trabajan por ejemplo, por un salario mínimo. Otra cosa es no estar recibiendo pensión y subsidio de otra índole del Estado. Otro de los requisitos es que la persona viva acá, que no tenga pensión. Que no tenga redes de apoyo sólidas: hijos y demás que solventen de alguna manera su situación económica y personas que no tengan predios. Persona víctima de cualquier forma de violencia, persona mayor de edad de entre todos los solicitantes, persona mayor con niños, niñas, jóvenes, personas con discapacidad o personas mayores que dependen económicamente de él. Persona mayor remitida por instancia como: Defensoría de derechos humanos, Personería, Contraloría, se debe priorizar. Los enviados por cualquier ente territorial. Otro de los criterios es que pertenezcan a grupos étnicos, afro, raizales, indígenas y población LGTBI. (Reconstrucción a partir de algunas de las entrevistas realizadas en las diferentes localidades en 2013 y 2014)

Como se expone en las entrevistas realizadas, para brindar servicios como los subsidios, se establecieron inicialmente unos criterios de focalización. Sin embargo, la PPSEV actual apunta a garantizar los derechos de todos los adultos mayores del Distrito y no únicamente los de personas con régimen subsidiado o que no tengan pensión. Con el enfoque de desarrollo humano se puede atender a todo adulto mayor que quiera recibir algún tipo de formación y no solo a quienes están en mayor condición de vulnerabilidad. Aun cuando la política plantea la atención a toda la población, se necesitan reformas en el sistema de ingreso de las personas -enfocado inicialmente al régimen subsidiado-y una mejor definición de criterios para ingresar a los diferentes servicios ofrecidos.

Anteriormente sí había unos criterios muy de focalización, por ejemplo nivel de Sisben, pero con el enfoque de derechos, lo que se busca es tratar de mirar cómo se llega al máximo de la garantía de derechos a toda la población. Entonces lo que se hizo fue generar unos criterios de participación que son muy generales. Si tú los miras dirías: ahí entra todo el mundo, pero para filtrar un poco, porque sabemos que los recursos son escasos, se determinan unos criterios 
de priorización y esos ayudan a ir intencionalmente focalizando un poco quiénes deberían estar o no en el servicio. Aunque lo que se quiere romper es eso. "No focalicemos porque estamos en un enfoque de derechos, se supone que no debemos focalizar". Debemos tratar de llegar a la población que realmente lo necesite. Entonces cuando tú preguntas acá, población focalizada, pues difícilmente, más bien población atendida es muy clave. (Reconstrucción a partir de entrevista realizada en el marco de la investigación en 2014)

El registro más general que lleva cada una de las localidades es la población atendida, si se tiene el dato sobre la población total de adultos mayores, sería posible establecer la cobertura real del programa. En la siguiente tabla se relaciona la población atendida por localidad con subsidio económico, no se incluye la atendida en los tres centros (Centro día, Centro noche y Centro de protección):

Tabla 1. Secretaría Distrital de Integración Social (SDIS). Proyecto 742. Servicio social, desarrollo de capacidades y potencialidades con apoyo. Diciembre 2013

\begin{tabular}{|l|l|l|l|l|l|}
\hline \multicolumn{1}{|c|}{ Localidad } & $\begin{array}{c}\text { Apoyo } \\
\text { Económico A }\end{array}$ & $\begin{array}{c}\text { Apoyo } \\
\text { Económico B }\end{array}$ & $\begin{array}{c}\text { Apoyo } \\
\text { Económico B } \\
\text { Desplazado }\end{array}$ & $\begin{array}{c}\text { Apoyo } \\
\text { económico D } \\
\text { cofinanciado }\end{array}$ & Total \\
\hline Usaquén & 6 & 1180 & 7 & 915 & 2108 \\
\hline Chapinero & 4 & 378 & 0 & 116 & 498 \\
\hline Santafé & 36 & 2186 & 30 & 1252 & 3504 \\
\hline San Cristóbal & 50 & 2733 & 20 & 3279 & 6082 \\
\hline Usme & 35 & 2807 & 127 & 3162 & 6131 \\
\hline Tunjuelito & 20 & 1191 & 9 & 1025 & 2245 \\
\hline Bosa & 44 & 3669 & 45 & 3225 & 6983 \\
\hline Kennedy & 34 & 2977 & 58 & 2282 & 5351 \\
\hline Fontibón & 8 & 774 & 16 & 418 & 1216 \\
\hline Engativá & 21 & 2161 & 45 & 2206 & 4433 \\
\hline Suba & 28 & 2075 & 36 & 1692 & 3831 \\
\hline Barrio Unidos & 7 & 671 & 4 & 248 & 930 \\
\hline Teusaquillo & 2 & 183 & 0 & 4 & 189 \\
\hline Mártires & 18 & 921 & 15 & 222 & 1176 \\
\hline Antonio Nariño & 8 & 247 & 1 & 244 & 500 \\
\hline Puente Aranda & 7 & 562 & 2 & 86 & 657 \\
\hline Candelaria & 1 & 258 & 1 & 0 & 260 \\
\hline Rafael Uribe & 20 & 3179 & 23 & 2731 & 5953 \\
\hline Ciudad Bolívar & 30 & 4732 & 156 & 4718 & 9636 \\
\hline Sumapaz & 1 & 73 & 0 & 0 & 74 \\
\hline Distrital & 0 & 10 & 0 & 0 & 27825 \\
\hline Total & 380 & 32967 & 595 & & \\
\hline Fuente & & & & \\
\hline
\end{tabular}

Fuente: Subdirección para la vejez (2012) 
Un segundo grupo de servicios corresponde a los Centros de atención (día, noche y protección social). El Centro día es una:

[...] prestación social de atención integral durante el día diseñado para personas mayores en condiciones de vulneración o amenaza en su integridad que requieran acompañamiento social para estimular procesos de autonomía, además de promover el ejercicio de los derechos en el marco de la seguridad humana”2. (Secretaría Distrital de Integración Social, 2014)

Este tipo de asistencia se encuentra dirigido a adultos mayores que no necesitan del subsidio económico pero que están interesados en la formación que se ofrece en desarrollo humano dentro de estas instalaciones.

Hay una población muy pequeña, que se acerca a la Secretaría por proyectos donde pue-
dan hacer uso de su tiempo libre, ċsí? Por eso es que acá se tiene un proyecto, es decir la
localidad, Secretaría de Integración Social y la Alcaldía, para realizar un Centro día; que es
otro de los servicios que tiene la Secretaría de Integración Social. Se le brinda atención a las
personas, no necesariamente por que tengan o porque necesiten un apoyo económico, sino
porque tienen otro tipo de necesidades. Es el de atención a personas, no necesariamente
con apoyo económico. Es un lugar de capacitación en desarrollo humano, recreación, lú-
dica, etc. Porque la base de la Bogotá Humana es el desarrollo humano. (Reconstrucción a
partir de algunas de las entrevistas realizadas en las diferentes localidades en 2013 y 2014)

En el caso del Centro día se presenta uno de los servicios más recientes de la subdirección de vejez que trata de superar el enfoque asistencialista aún vigente en la entrega de subsidios económicos, con actividades de formación y lúdicas. La administración del plan de desarrollo distrital "Bogotá humana" ha hecho gran énfasis en la importancia de la formación, la autonomía y el desarrollo de capacidades y potencialidades.

El Centro Noche es un servicio de carácter transitorio que ofrece atención integral en busca de garantizar un alojamiento seguro para personas mayores de 60 años que no cuentan con un domicilio permanente para pasar la noche. Pretende garantizar la restitución de derechos a través de la satisfacción de necesidades básicas como dormitorio, alimentación y actividades de desarrollo humano que promuevan un envejecimiento activo. (Secretaría Distrital de Integración Social, 2014).

\footnotetext{
${ }^{2}$ Aunque es una definición tomada de la página de la Secretaría de Integración Social, el Servicio está reglamentado por el Art. 2 Ley 1315 de 2009.
} 
Un Centro noche es donde las personas mayores pueden irse a quedar a dormir, pero si por ejemplo, se está en Centro día, no se puede estar en Apoyo económico. No puede haber simultaneidad entre los servicios. (Reconstrucción a partir de entrevista realizada en el marco de la investigación en 2014)

Por último, el Centro de protección social es un servicio dirigido a personas mayores de 60 años con un grado de dependencia alto o severo, sin redes familiares o sociales de apoyo, en situación de fragilidad y vulnerabilidad social. En este se llevan a cabo acciones integrales interdisciplinarias en el marco de los enfoques de derecho y desarrollo humano, prestando un servicio continuo las 24 horas, de domingo a domingo (Secretaría Distrital de Integración Social, 2014) ${ }^{3}$.

Aunque visto desde afuera este servicio parece muy llamativo para la población más vulnerable, no es atractivo para el adulto mayor, ya que al internarse, lo hace a tiempo completo y pierde las demás actividades que podría realizar estando en la calle. En ese sentido, internarse no es una opción independientemente de su vulnerabilidad, al ser más significativa la pérdida del 'tiempo libre'.

Como estamos liderando la política de vejez y envejecimiento y estamos haciendo la protección a los derechos de las personas mayores, tenemos dos Centros de protección para la persona mayor, que son transitorios: Bello Horizonte que queda en San Cristóbal y el de Bosque Popular, que queda al lado del Club de Compensar. Las personas que llegan allá son personas absolutamente solas, necesitan enfermero casi durante las 24 horas. Les dan su cama, les dan su comida. Todos sus derechos están protegidos dentro de esos sitios. Son transitorios, mientras la persona se acomoda a estar ahí. No es fácil, la mayoría de personas no lo eligen porque no los dejan salir.

La Secretaría además, tiene otros convenios con otras instituciones que sí son hogares geriátricos-gerontológicos, para que estas personas, después del proceso de armonización en estos sitios, se puedan ir a esos centros geriátricos. Aunque están en otras localidades, no quiere decir que San Cristóbal tenga solo personas de San Cristóbal no, se ubicó allí por las instalaciones y por el espacio físico, el Centro de protección Bosque Popular igual. Y luego ellos son remitidos a otros centros de protección fuera de la ciudad. Entonces Secretaría les tiene uno en Fusagasugá y otro en Girardot por el clima y nosotros hacemos la supervisión de todos los centros geriátricos-gerontológicos de la ciudad. Para el ingreso de la persona a este servicio hacemos todo el trámite con los centros de protección de la Secretaría donde pueden recibir al adulto mayor, hacemos validación de condiciones con

\footnotetext{
${ }^{3}$ Aunque es una definición tomada de la página de la Secretaría de Integración Social, el Servicio está reglamentado por el Art. 2 Ley 1315 de 2009.
} 
los hospitales para mirar su situación y se toma la medida de protección. (Reconstrucción a partir de algunas de las entrevistas realizadas en las diferentes localidades en 2013 y 2014)

Vamos a construir un Centro de protección en la zona rural, hay once personas en completo abandono y se considera prioritario que esas personas estén institucionalizadas debido también al sentido que ellos le han dado a su vida en lo rural. Entonces la Secretaría, junto con la Alcaldía y con la Secretaría de Salud, se dio a la tarea de iniciar el proceso. (Reconstrucción a partir de entrevista realizada en el marco de la investigación en 2013)

\section{Los servicios y el enfoque de desarrollo humano}

$\mathrm{Al}$ ser este es el eje transversal que fundamenta los servicios que presta la Subdirección para la vejez, es importante generar una sección propia para esta oferta institucional que no aparece en la Secretaría de Integración. En el caso de los subsidios, que es el más difundido en las localidades, se procede con talleres de formación en desarrollo humano. Estos parten de un direccionamiento dado en el nivel central, pero se aplican de acuerdo a lo establecido por cada subdirección local.

El objetivo de esos encuentros es que a través de esos espacios de reflexión, los adultos mayores logren entender ciertas cosas que tienen que ver con su vida. Que ellos entiendan que hay una responsabilidad consigo mismos a pesar de su edad, que existe un entorno, que hay personas con las que ellos también tienen responsabilidad en lo social. Uno les hace entender que son personas capaces, que tienen potencialidades, que tienen muchas formas de explotar esas cosas que ellos tienen, desarrollar en ellos un sentido de pertenencia frente a su entorno. Trabajamos qué significa ser un ciudadano, les hablamos por ejemplo de educación sexual, que es un tema que a veces parece loco tratar con adultos mayores pero que es necesario. Digamos que ahí nos apoyamos mucho en instituciones locales, por ejemplo ahí el IDRD nos ha ido apoyando. Además, desarrollo humano tiene líneas: una primera que es un grupo de priorización, ya tienen una estructura clara en términos de metodología, temáticas, etc. Pero aquí viene más gente para desarrollo humano, diferente al grupo de priorización. Aquí nosotros no reportamos meta, pero igual las personas se capacitan y reciben talleres y demás. (Reconstrucción a partir de algunas de las entrevistas realizadas en las diferentes localidades en 2013 y 2014)

Los encuentros de desarrollo humano funcionan de manera diferente dependiendo de la localidad. Sin embargo, el objetivo es similar: brindar talleres que le otorguen a las personas mayores nueva información para fortalecer sus capacidades y potencialidades, orientándose hacia una autonomía política y social que les permita convertirse en ciudadanos activos, dados a movilizarse en pro de sus derechos, pero asumiendo las responsabilidades del caso. 
Los talleres apuntan a dos poblaciones que pueden tener objetivos diferentes al asistir a estos espacios. Para aquellas personas que reciben apoyo económico, la asistencia al taller garantiza la entrega del subsidio, mientras que hay un grupo poblacional de pensionados que van a los talleres a recibir la información, únicamente por gusto. Sin embargo, medir el grado de compromiso de los asistentes al taller con su formación sigue siendo un reto que Integración Social deberá cumplir.

Aunque desde el nivel central aún no se ha dado un marco conceptual suficiente acerca de cómo debe entenderse el desarrollo humano, se brindan líneas temáticas que cada líder de proyecto desarrolla bajo su propia lógica y con su equipo de trabajo en los talleres. Este le apunta a las particularidades de su localidad, ya sea disponiendo a un único profesional para desarrollar los talleres dentro de las locaciones de la subdirección o coordinando a todo el equipo que apoya el proyecto para la elaboración y ejecución de los talleres en cada Unidad de Planeamiento Zonal (UPZ). En general, los talleres apuntan a satisfacer las dimensiones de la PPSEV en todas las localidades. Es decir que se centran en restablecer y garantizar los derechos de las personas mayores.

La primera o segunda semana del mes nos organizamos para los talleres de encuentro de desarrollo humano. Que son en las diferentes UPZs. Entonces en los diferentes salones comunales se hace la convocatoria para que las personas mayores no se desplacen hasta acá, sino que se queden en los diferentes territorios, entonces ellos asisten a esos encuentros de formación. La mayoría tienen ese objetivo de que no les ponga la falla porque si tienen más de dos fallas se les bloquea el subsidio. (Reconstrucción a partir de entrevista realizada en el marco de la investigación en 2013)

\section{Fortalezas y debilidades de la implementación de la PPSEV}

Habiendo explicado los servicios que presta la Secretaría además de otros conceptos necesarios para entender cómo se operacionaliza la política en las diferentes localidades, a continuación se evidencia - a través de las entrevistas- cuáles han sido las principales fortalezas y debilidades en el modo de proceder desde cada territorio, incluyendo un balance de los servicios, el trabajo intersectorial, la territorialización, la conformación de los equipos de trabajo, entre otros.

\section{Servicios}

El mayor avance que han tenido los servicios ha sido la anexión del componente formativo en derechos humanos, cuya implementación pretende superar el enfoque asistencialista que conserva el subsidio. Hoy en día, aun cuando se brinda un servicio de apoyo económico indispensable para las poblaciones más vulnerables -dada la violación de sus derechos económicos y la deuda social que afronta el resto de la ciudad para con 
ellos- se intenta incorporar talleres de desarrollo humano como un mecanismo que, además de permitir el seguimiento de los adultos mayores que asisten a los encuentros, les brinda una mayor calidad de vida a nivel social y cultural. Los talleres apuntan a dar a conocer información importante para la población y a tratar de cuestionar los imaginarios negativos sobre la vejez. Aunque hay un avance en estas tareas, es necesaria una articulación más fuerte con otro tipo de instituciones en aras de lograr el objetivo de la PPSEV. En ese sentido, los servicios se vinculan directamente con las dimensiones de la política, respaldando el principio fundamental de la misma: la dignidad humana.

Creo que la entrega de esos recursos ha contribuido a que muchos adultos mayores en situaciones en donde no tienen qué comer, no tienen cómo pagar su arriendo, su habitación de alguna manera, soluciona parte de sus problemas con esos $\$ 120.000$ pesos. Aunque es algo muy mínimo para la cantidad de necesidades que ellos tienen, es una forma de que ellos tengan autonomía y también les ayuda un poco al proceso de socialización con un par. Hay personas que entran al proyecto, son vecinos y hasta que entran se hablan, y como ellos mismos dicen, para compartir soledades. (Reconstrucción a partir de algunas de las entrevistas realizadas en las diferentes localidades en 2013 y 2014)

En lo que ha avanzado la institución es en lograr que ellos se vinculen en varios servicios al mismo tiempo cuando están en vulnerabilidad extrema. Los servicios no se dan al azar. En algún momento era una cosa así. No, nosotros vamos y confrontamos la situación. Evidenciamos que sí, que efectivamente se necesita. (Reconstrucción a partir de entrevista realizada en el marco de la investigación en 2014)

Creemos que hay muchos adultos mayores que ya disfrutan esos espacios de desarrollo humano. La gente viene y pregunta: nos gustaría recibir curso de no sé qué cosa o cuándo van a empezar los cursos y eso nos dice que la gente ya se lo está tomando un poco más en serio. La idea es que este año, con la nueva propuesta de desarrollo humano podamos por ejemplo, hacer salidas pedagógicas, hacer recorridos históricos por el centro de Bogotá, porque estamos hablando de ciudadanía, tenemos que ponernos en contexto. La idea es ver al adulto mayor de una manera distinta, no es solamente dar un subsidio, sino reconocer que es un ser humano con unas necesidades. Yo creo que la misión cuando uno trabaja con población como esta, tiene que trascender lo económico y solventarle el día a día. Es cambiar percepciones, generar nuevas sensaciones, nuevas experiencias con relación a su vida y la etapa en la que está. (Reconstrucción a partir de entrevista realizada en el marco de la investigación en 2014)

Los servicios sociales que han nacido posteriores a la política, obviamente tienen una mayor claridad hacia dónde dirigir sus acciones. Se está avanzando en tratar de que dejen de ser prestacionales y estén centrados en poder posicionar a las personas mayores como sujetos de derechos. Lo digo por el ejercicio que hicieron en Centro día, hay obviamente unos mínimos de entorno que se brinda allá, pero hay una motivación más allá de "qué 
me van a entregar", lo que ha sido más difícil con servicios que llevan muchos años, donde ya hay ligado un factor económico, que está presente en poblaciones tan vulnerables. (Reconstrucción a partir de entrevista realizada en el marco de la investigación en 2014)

Adicionalmente se ha progresado en la forma de asignar cupos en las localidades con el uso de criterios técnicos para tomar la decisión y no guiados solamente por tamaño de la localidad.

Para esa asignación de cupos se hizo un ejercicio ya teniendo en cuenta varias variables. Sé que se trabajó con el equipo de subsidios y de ahí se determinaron los cupos de cada localidad. Ya hay un criterio para esa distribución. Eso también ha servido para que los nuevos servicios, por decir Centro día, tienen una meta distrital, pero no podría ser tan aleatoria que estuviera puesto el servicio social en una localidad $\mathrm{x}$ sino que empezaron a cruzarse una serie de variables, en eso digamos que se ha avanzado. (Reconstrucción a partir de entrevista realizada en el marco de la investigación en 2014)

Respecto a las debilidades que presenta la implementación de la PPSEV se reconoce tanto en las localidades como a nivel central, una limitación de recursos. Esto no permite que haya un reconocimiento de la seguridad económica como derecho, ni para la población que ya recibe el servicio, ni para aquellas que son potenciales aspirantes. Especialmente en el caso de los subsidios entregados a las personas mayores más vulnerables, dado que para muchos de ellos que no poseen ninguna otra fuente de ingreso, el monto es muy bajo.

El servicio siempre lo he visto como restituirle esos derechos que el Estado no les dio a las personas mayores en su momento. Sin embargo, ellos mismos los llaman 'subsidios de miseria'. Cuando uno termina de hacer la cuenta de cuánto gasta una persona, mensualmente no da. Ellos tienen que rebuscarse. A veces tenemos que conformar una red institucional más fuerte que le garantice su bono para que pueda pagar el arriendo. Tenemos que hacer una protección tan fuerte, porque los servicios a veces están limitados en eso, que son muy pequeños. Otra dificultad es que los cupos de los subsidios son limitados. Nosotros tenemos en este momento represadas personas que ameritan la entrega de ese subsidio, pero no hay cupos, porque no hay recursos. O sea, los recursos de alguna manera, desde la Presidencia, el Distrito y la localidad, no alcanzan a cubrir las necesidades y las solicitudes de las personas. La demanda es muy grande y en términos de recursos económicos, ni el Distrito, ni los recursos que vienen directamente de Presidencia alcanzan a cubrir las necesidades de subsidios que nosotros tenemos. (Reconstrucción a partir de algunas de las entrevistas realizadas en las diferentes localidades en 2013 y 2014) 
Aun cuando los servicios implementados representan grandes avances en la atención de las necesidades de las personas mayores, el servicio que más tiempo lleva es el de apoyo económico. Al tener un carácter de auxilio, mantiene una connotación asistencialista en el imaginario de las personas que lo reciben no obstante la intención de SDIS por cambiar este imaginario. Inicialmente para su entrega solo se necesitaba ir a firmar la asistencia en las subdirecciones. Se ha intentado cambiar esta modalidad con la creación de los talleres de desarrollo humano inspirados en el Plan de Desarrollo "Bogotá Positiva” 2012-2016. Es por esto que la superación de esa visión es todavía uno de los retos más importantes que debe afrontar la PPSEV.

Las personas ven el subsidio como una obligación y no se comprometen con su formación. La entrega de este está ligada a que ellos asistan a los cursos de desarrollo humano y eso rompe con el objetivo. La idea es que las personas estén allí porque reconocen que hay un compromiso con ellos mismos, con su entorno, y no solamente por la entrega de un subsidio económico. A veces siento que la gente siente que es una obligación del Estado. Entonces creo que la entrega de los subsidios debería tener otro objetivo que no sea solamente eso. (Reconstrucción a partir de entrevista realizada en el marco de la investigación en 2014)

Uno pensaría que todos los servicios de alguna u otra manera deberían tener un componente de formación ciudadana, porque al fin y al cabo muchas veces las personas llegan incluso a pedir una cita médica o un servicio recreativo y piensan que eso es un favor o que es una limosna. Entonces ahí hay vacíos y pues eso vacíos van en armonía con lo que pasó en el proceso de formulación. (Reconstrucción a partir de entrevista realizada en el marco de la investigación en 2014)

La política dice que hay que crear vínculos familiares, ¿sí? Eso sería chévere y uno de profesional trabaja los vínculos, digamos con un taller y ya, que cree vínculos no, eso no es fácil cuando uno ve la realidad de su territorio. Esa realidad donde el costo de vida es muy alto, donde a veces los hijos no tienen una estabilidad laboral, cuando hablamos de derecho al trabajo, ¿̇sí? La persona mayor debe tener derecho al trabajo y eso no se está garantizando y pueda que todos estemos trabajando en función de esto, pero en la realidad no se está dando. (Reconstrucción a partir de entrevista realizada en el marco de la investigación en 2013)

En el caso de los otros servicios el no poder cubrir a todas las personas que necesitan del recurso podría verse como una falta a la política. Una solución podría ser la articulación con instituciones no gubernamentales que estén interesadas en la protección y formación de los adultos mayores.

El número de personas no debería ser un condicionamiento para montar el centro de protección Centro día, porque existe la necesidad en el territorio. Pero al momento de priorizar 
recursos se descuida esa población y se va en contravía de los objetivos de la política. Garantizar el derecho a... y más a un artículo constitucional que dice que las personas mayores merecen especial protección del Estado, entonces otra limitación ha sido esa. (Reconstrucción a partir de entrevista realizada en el marco de la investigación en 2014)

Una de las inquietudes que surge, además de la falta de recursos económicos que presenta la ejecución de la PPSEV, está en los recursos físicos. Parece que algunos de los objetivos de la política superan los recursos y alcance de las actividades que solamente puede desarrollar la Subdirección para la vejez. Hay cambios de imaginarios que solo pueden lograrse en el mediano y largo plazo involucrando tanto a adultos mayores como a la comunidad en general. Los cambios institucionales por su parte, solo podrían lograrse bajo la articulación de instituciones gubernamentales, no gubernamentales, sociedad civil y con la suficiente voluntad política. Los talleres de desarrollo humano, aunque constituyen un avance, son apenas un primer paso para lograr cambios sociales profundos.

\section{Trabajo intersectorial}

Otro de los principales avances observados es la articulación de la Subdirección con otras instituciones que tienen una responsabilidad directa con la PPSEV, como la Secretaría de Salud, los hospitales e incluso los operadores de las demás políticas. También hay localidades que se han aliado con instituciones educativas o incluso con la misma sociedad civil. Lo anterior supone un adelanto dado que estos trabajos conjuntos no se hacen bajo ningún compromiso vinculante sino partiendo del interés particular de cada entidad por responderle a la población.

En infancia hicimos toda una capacitación de los profesionales de este equipo con los profesionales de infancia, para lograr describir situaciones y conformar los conceptos técnicos en visitas domiciliarias. Como ámbito familiar, va a todas las viviendas del sector. Si identifica una persona mayor, como ya tiene nuestra línea técnica, construye descripciones y conceptos que nos favorecen. Se ingresa a esa persona al servicio sin invadir el espacio de ellos. LGTBI se ha articulado con nosotros también. Los referentes van, trabajan con las personas mayores. Hace un año nos gritaban cuando hablábamos de LGBTI: “iesto es Sodoma y Gomorra!” Ya ahora por lo menos escuchan, entienden. Hemos avanzado con que se sientan orgullosos de que sus hijos asumieron ese reto ante la sociedad, de verse como se sienten. (Reconstrucción a partir de entrevista realizada en el marco de la investigación en 2013)

Asimismo el trabajo con dinamizadores es importante por el vínculo que establece con los adultos mayores como sujetos autónomos que cuidan de otros, mostrando que el éxito en la gestión de la articulación, muchas veces tiene que ver con las personas 
mayores que lideran la iniciativa en cada localidad. Lo mismo sucede con el trabajo realizado con las instituciones educativas.

Nosotros no podemos hacer un manejo tan apropiado por los quehaceres. Entonces nosotros tenemos delegadas a unas personas mayores que son de nuestro territorio que los llamamos dinamizadores. Son personas que les gusta estar activas, participar en reuniones. Ellos nos colaboran con las visitas, conocen el territorio, saben dónde vive zutanito, fulanito. Ellos nos pasan un informe mensual de la persona: cómo se encuentra, cómo está su estado de salud, si está viva. Pues ese es un seguimiento y cada compañero le hace reconocimiento a sus dinamizadores, porque no es una labor que se les pague, ellos lo hacen como algo voluntario. (Reconstrucción a partir de algunas de las entrevistas realizadas en las diferentes localidades en 2013)

En este momento hay un programa muy interesante con algunas universidades, tiene que ver con las instituciones que no son del Distrito. Tenemos estudiantes que hacen algunas pasantías acá y que apoyan todo el tema de los encuentros en desarrollo humano. (Reconstrucción a partir de entrevista realizada en el marco de la investigación en 2014)

Sin embargo, estos esfuerzos siguen centrados en dos o tres entidades. La débil coordinación entre las instituciones gubernamentales y no gubernamentales limita la adecuada operacionalización de la política. Sin una cooperación de todos los sectores es muy difícil restaurar e incluso garantizar los derechos de las personas mayores, haciendo imposible adoptar las dimensiones de la política. La Secretaría de Integración, así como las demás instituciones, tiene recursos limitados que si se coordinaran podrían brindar un mejor apoyo a la población mayor del distrito y una cobertura más amplia.

Como quien lidera las políticas públicas es Integración Social, prácticamente nos están dando toda la responsabilidad a nosotros. Construir esos planes de acción de acuerdo a lo que solicita el Decreto fue un despliegue de Integración Social y prácticamente fue ejercicio puerta a puerta. Identificamos que Hábitat tiene vivienda, identificamos que Secretaría de Desarrollo Económico debe generar iniciativas, pero cuando vemos que ellos no lo están haciendo, pues nosotros nos quedamos cortos y no damos respuesta efectiva a la comunidad. Aunque sale un Decreto, los sectores aún no se han comprometido. (Reconstrucción a partir de entrevista realizada en el marco de la investigación en 2013)

A nivel distrital hay algunos sectores que creen que ellos no tienen nada que ver con la política pública, no se sienten tocados. Entonces a veces ellos dicen: pero yo que tengo que aportar. No tienen la dimensión de lo que plantea la política y entonces en esa línea no tienen referentes de política o tienen un referente para muchas políticas entonces no se va a centrar en una. Eso debilitó la misma construcción de los planes de acción, porque 
si bien son institucionales, casi que tocó con tirabuzón sacar información, fue muy difícil y eso que está por Decreto. Inicialmente salió una circular pero después lo elevaron a Decreto, para hacerlo obligatorio y a pesar de eso hay dificultades. (Reconstrucción a partir de entrevista realizada en el marco de la investigación en 2014)

\section{Territorialización}

Los avances particulares de ciertas localidades en el proceso de territorialización han estado ligados a las iniciativas de los equipos de trabajo que allí operan, más que por los lineamientos dados desde el nivel central.

Yo inicié como referente de cobertura, no era líder del servicio. Entonces iniciamos con 280 personas más o menos activas. Cuando desde la Alcaldía llega la directriz que hay unos territorios que se tienen que priorizar y si ustedes van a ver la caracterización, tenemos un porcentaje elevado en ciertas UPZ, en esta parte donde se puede caminar, que son las de mayor cobertura. Pero irse por allá arriba donde llega uno al tope con Chipaque ya pues, no se había priorizado, hacer el recorrido por las veredas tampoco se había priorizado. Entonces el año pasado trabajamos en eso. Logramos que la lista de espera disminuyera. (Reconstrucción a partir de entrevista realizada en el marco de la investigación en 2013)

Sin embargo, una de las dificultades experimentadas en las localidades es dar a conocer la existencia de la PPSEV a la población objetivo. Unas localidades han avanzado más en este aspecto. La territorialización sigue presentando dificultades, tanto por falta de personal para llegar a las distintas UPZ de las localidades, como por falta de recursos para los desplazamientos en las localidades. Las zonas rurales experimentan mayores dificultades para que su población acceda a los servicios, tanto por su connotación rural, como por la gran distancia del centro urbano.

Dificultad de acceso porque es una localidad muy grande. Entonces que las personas logren conocer los servicios es un reto, que las personas se inscriban en los servicios, es un reto. Tenemos dificultad en insumos, para que los servicios operen. Entonces no tenemos transportes, nosotros tenemos que usar nuestros propios medios, no contamos con seguridad. (Reconstrucción a partir de entrevista realizada en el marco de la investigación en 2013)

Un tema que queremos desarrollar en desarrollo humano, es el de la ruta de atención a adultos mayores, que no existe y sí existe, es absolutamente invisible. Ellos no saben qué hacer cuando se sienten maltratados, a dónde pueden ir, a dónde pueden denunciar. Queremos que reconozcan que ellos también pueden hacer valer sus derechos y que hay unos mecanismos ciudadanos que les van a permitir hacer restauración de derechos. En eso nos está apoyando el Hospital de Chapinero, porque ellos han venido diseñando un programa muy interesante 
con relación a esto. Reconozco que nosotros deberíamos tener estrategias en territorio mucho más puntuales para contarle a la gente qué tipo de servicios tenemos, lo que pasa es que como no tenemos tanta cobertura, ahora el equipo se nos puede salir de las manos. (Reconstrucción a partir de entrevista realizada en el marco de la investigación en 2014)

\section{Equipos de trabajo en las localidades}

Respecto a esta área, la subdirección ha empezado a cualificar a los grupos de trabajo en cada subdirección local, contratando personal capacitado de acuerdo a las necesidades del equipo y sus funciones.

Nosotros nos organizamos de tal forma que hemos logrado cumplir las metas de manera transparente, hemos trabajado en equipo y no hemos cumplido metas como por cumplir, porque hay un equipo comprometido, en formación permanente. Pero en otras localidades la dificultad que se ve es esa: la falta de organización y de compromiso de los servidores públicos. Conocer la finalidad del servicio o sea, qué es eso, apropiarse de la política pública y eso es lo primero que uno tiene que hacer cuando trabaja en esto. (Reconstrucción a partir de entrevista realizada en el marco de la investigación en 2013)

En la Subdirección hemos tratado de ser muy cuidadosos para garantizar que la persona que está al frente sea la persona idónea y que cumpla ciertas características para ejercer, o bien el rol de liderazgo de política o el rol de liderazgo de un servicio social. Además se han venido vinculando otro tipo de profesiones que antes no eran contemplados, como los artistas, en relación con las otras políticas es algo que se está visibilizando. La Subdirección para la vejez tiene unos equipos estructurados a nivel local. Inicialmente los líderes en su momento estuvieron muy centrados en el tema de subsidios a nivel local, ahorita la mirada y el acompañamiento que se les está dando es desde el de líder del proyecto, más que desde el servicio y deben tener la mirada estructural del tema en la localidad. Se busca que puedan identificar no solo cuántas personas mayores hay sino cómo está el tema de vejezy envejecimiento en la localidad. (Reconstrucción a partir de entrevista realizada en el marco de la investigación en 2014)

Sin embargo, como ya se ha mencionado, la forma de proceder respecto a la prestación y territorialización de los servicios corresponde al manejo de cada líder de proyecto en su localidad y sus posibilidades, lo que genera desarrollos distintos. En este sentido, es necesario imaginar mecanismos que permitan equilibrar los esfuerzos personales con los institucionales.

\section{Trabajo intergeneracional}

Aunque en la política ya se reconoce la importancia de entender la vejez en el contexto del envejecimiento como un proceso biológico que inicia con el nacimiento y finaliza 
con la muerte, el trabajo de la política se ha enfocado en los adultos mayores. Lo cierto es que esta es una condición de la que todo ser humano debe ser consciente para poder responder a ella de manera óptima, tratando de minimizar las vulnerabilidades características de dicha etapa. En el trabajo con la comunidad, es con las familias donde se puede empezar a considerar el cambio de imaginarios sobre la vejez. Este tipo de procesos tiene resultados identificables a largo plazo, es por ello que los avances posiblemente se den tan solo hasta la siguiente generación.

Aún queda como reto para la ciudad lograr una articulación tal de las políticas poblacionales que permita mejorar el bienestar conjunto de los ciudadanos, quienes finalmente son un solo individuo que pasa por todas las etapas del ciclo vital y por tanto, por cada política.

\footnotetext{
Eso es un trabajo que nosotros tenemos que hacer porque no se ha hecho. O sea, de cierta manera está segregado. Aquí tenemos adulto mayor, aquí tenemos familia, infancia, pero no hay como una estrategia clara que permita de alguna manera buscar un punto de encuentro con todas las poblaciones. Creo que es un trabajo que sabemos que toca hacer, pero no se ha hecho. Las poblaciones están sueltas y cada una en sus dinámicas. Parte de la discusión es que para cada una hay una política, ¿no? Y la discusión es esa, que no hay una conexión entre política y política, pero es un tema que se ha discutido en mil escenarios y que el Distrito sabe que tenemos que hacer, pero no hay un trabajo claro con relación a eso. (Reconstrucción a partir de entrevista realizada en el marco de la investigación en 2014)
}

\section{Seguimiento y monitoreo}

Aunque en el 2009 el Centro de Investigación para el Desarrollo (CID) de la Universidad Nacional presentó la línea base, los funcionarios a nivel local no tienen claridad sobre cómo está avanzando el tema o qué indicadores adicionales al número de personas atendidas en el subsidio deberían llevar. A nivel central son conscientes de la necesidad de obtener indicadores de evaluación y de impacto que midan el desempeño real de la política en el territorio. Aún cuando en algunas localidades se percibe un cambio positivo en la población, es importante tener una medición efectiva. Adicionalmente, las cargas de trabajo que se manejan en todas las localidades no permiten que los profesionales que van a territorio ni aquellos dedicados al área administrativa se encarguen de hacer un adecuado monitoreo y seguimiento.

Realmente la gente, medirlo juiciosamente, eso no se hace. Aquí está limitado solo al tema de los números. Número de personas activas, número de personas egresadas del servicio, número de personas atendidas y número de personas que asisten al curso de desarrollo humano. Aquí es más o menos meta, meta cumplida. Yo sí creo que hay muchas cosas 
que, sobre todo en el tema de desarrollo humano, porque digamos que en subsidios, tú qué más puedes mostrar, simplemente número de personas que reciben, que ya no reciben, eso es clarísimo. (Reconstrucción a partir de entrevista realizada en el marco de la investigación en 2014)

Cuando se fijan esas acciones, tanto a nivel local como a nivel distrital, si tú miras también las matrices, nos vemos escasos de indicadores. De indicadores y de línea de base. Entonces seguimos haciendo acciones en el vacío, porque no sabemos efectivamente cómo está la situación de las personas mayores. Tú miras una matriz de esas y las columnas que menos se diligenciaron fueron esas dos. ¿Cuál es la línea de base? Y en casi todas aparece, no aplica, porque creo que el problema no es solo con esta política sino con muchas cosas que se hacen. Además los instrumentos de seguimiento y monitoreo no están todavía, no nos los han entregado a nosotros los responsables de cumplir con esa información a nivel local. A nivel distrital han pedido una información de orden cualitativo y cuantitativo, que lo único que se hizo, fue mostrar avances frente a lo que se había hecho. Entonces todas esas actividades que tú conoces en lo local, todo eso se recoge, lo que han hecho los diferentes sectores como para aportar algo al cumplimiento de ese plan de acción, pero no hay un instrumento que permita mirar cuál es la trazabilidad que se está dando frente a Y o X problema. No hay una cuestión que uno diga, ¿qué había antes y qué impacto tuvo eso después? ¿Que pasó y hasta dónde eso está cumpliendo una meta del Plan de Desarrollo o una meta del proyecto?, eso no se tiene claro. (Reconstrucción a partir de entrevista realizada en el marco de la investigación en 2014)

\section{Aportes para seguir avanzando en la implementación de PPSEV}

Vistas las fortalezas y debilidades que enfrenta la implementación de la PPSEV se sugieren algunas opciones para mejorar el trabajo que se desarrolla actualmente desde las diferentes subdirecciones:

- Equipo de trabajo: Es difícil llevar un monitoreo y seguimiento de la política con la actual sobrecarga de los equipos de trabajo en las localidades. Se propone la ampliación del personal para la realización de esta tarea y la sistematización de la información recogida en las fichas de visita domiciliaria. La contratación de pasantías con las universidades podría ser útil para esta tarea.

- Articulación interinstitucional: Se necesita mayor articulación institucional para acatar las propuestas hechas en la política relativas a la reforma del sistema de salud. Un esfuerzo conjunto, así como una mayor priorización del tema por parte de los diferentes sectores institucionales, facilitarían y mejorarían la implementación. Como alternativa se propone un mayor contacto desde las localidades con las organizaciones no gubernamentales que trabajen temas relativos a la vejez y el envejecimiento tales como ONGs. 
- Desde los territorios hacia lo distrital: la implementación de la política es más efectiva cuando se trabaja desde las particularidades de cada territorio, respetando las condiciones sociodemográficas y culturales de los adultos mayores. En ese sentido el abordaje de la vejez y el envejecimiento debe ser diferente en cada localidad, haciendo que los lineamientos de trabajo se den desde lo local hacia lo distrital. Es en la cotidianidad y el día a día de los gestores y líderes de política que trabajan directamente en el territorio que surgen las problemáticas a trabajar en la política y las potencialidades de cada localidad. Es por ello que desde lo local y con el apoyo de la comunidad, se deben diseñar las estrategias que pueden funcionar para llegar a la población según sus características y necesidades. Desde el Distrito se puede hacer la socialización de las estrategias para mejorar la territorialización en cada localidad.

- Criterios de priorización: Los criterios de priorización utilizados para establecer qué tipo de población recibe el servicio, aun cuando pretenden ser más incluyentes, todavía mantienen por fuera a la población que no pertenece al régimen subsidiado. Estos criterios ofrecen beneficios al maximizar la distribución de un recurso escaso por la falta de presupuesto y personal (los servicios) pero también limita, dado que deja por fuera a población que puede beneficiarse de espacios como los talleres de formación en desarrollo humano, dados los lineamientos de la política. La propuesta es revisar y mejorar los criterios para poder ampliar la cobertura.

- Trabajo intergeneracional: La cuarta dimensión de la PPSEV Envejecer juntos y juntas plantea la necesidad de hacer un trabajo conjunto entre todos los grupos etarios para cambiar los imaginarios segregacionistas existentes sobre el envejecimiento y la vejez. No obstante no ha sido posible hacer un trabajo suficientemente integrado desde los talleres que ofrecen las subdirecciones, ya sea por las particularidades de los territorios o por la dificultad de articular las diferentes políticas poblacionales, puesto que desde las otras políticas no se ha priorizado el envejecimiento en tanto curso vital.

\section{Reflexiones finales}

Aún con las dificultades que enfrenta la implementación de la política, la experiencia de gestores, coordinadores y profesionales de la subdirección de vejez en el nivel central evidencia una coherencia moderada ${ }^{4}$ entre los servicios prestados y los lineamientos de la política. Por ejemplo, el servicio de apoyo económico responde a la dimensión de Vivir bien en la vejez que trata de brindar seguridad económica a la población atendida. Aunque se reconocen los avances en el aumento y diversificación de los bonos, también se evidencia que estos subsidios son insuficientes para garantizar una vida digna, con la

\footnotetext{
${ }^{4}$ Se refiere a que la PPSEV tiene un enfoque de derechos que trasciende ampliamente la perspectiva tradicional de los servicios.
} 
que puedan cubrir vivienda, alimentación, transporte, entre otros. Del mismo modo, los demás servicios responden a alguna o varias dimensiones y las temáticas trabajadas en los talleres de formación están siendo permanentemente articuladas con las dimensiones y derechos que se pretende garantizar.

Como todo proceso social, la implementación de la política evidencia las tensiones existentes entre los escenarios deseables para un envejecimiento activo y digno, y las condiciones reales en las que estamos envejeciendo. En esas tensiones se desarrollan las actividades cotidianas de atención a la población mayor de 60 años con avances y dificultades que revelan por una parte, el esfuerzo de los equipos de trabajo de la Subdirección para la vejez por operacionalizar la política a través de procesos de formación y territorialización que se orienten hacia la superación del enfoque asistencialista y la armonización con los objetivos de la PPSEV; y por otra el desafío que constituye para el Distrito realizar un trabajo articulado, coordinado y comprometido entre las instituciones gubernamentales y no gubernamentales que definen y ejecutan la distribución de los recursos y acciones que pretenden mejorar la calidad de vida de los mayores y sus entornos.

En el trabajo intergeneracional, la política tiene el desafío de imaginar una estrategia que suavice las vulnerabilidades de la población en las etapas anteriores a la vejez, para que en el futuro no se deba ampliar el uso de la asistencia económica y así, superar el ciclo de pobreza en las generaciones futuras a través de la ampliación del acceso al empleo y la jubilación.

Uno de los retos más interesantes es el cambio de orientación en la forma de ver y atender la vejez que surgió hace más de un siglo con la creación de la Beneficencia de Cundinamarca. Esta institución respondía a unas condiciones de vida en las que la vejez se interpretaba como un estado de carencia física y social. La representación conservó su fuerza e influencia en la vida social de la ciudad a lo largo del siglo XX y se consolidó en la década de 1960 con la creación del Departamento Administrativo de Protección Social (SDIS, 2009, p.7) que realizaba su trabajo con personas mayores a través de la Oficina de Higiene Mental y Emergencia. Tal enfoque se topó con profundas contradicciones en la década de 1990 con la perspectiva de derechos que entiende la vejez como un estado funcional, física y socialmente. En este sentido el esfuerzo que viene realizando la Secretaría es más reciente de lo que se suele creer y requiere un trabajo integral y de largo plazo que vincule, no solo a las instituciones sino a la población en su conjunto para estimular la modificación de la forma en la que históricamente se han relacionado las personas mayores con la sociedad.

\section{Referencias}

Acaldía Mayor de Bogotá (2010). Política Púlica Social para el Envejecimiento y la Vejez en el Distrito Capital 2010-2025. Bogotá: Alcaldía Mayor de Bogotá. 
Alcaldía Mayor de Bogotá (2012). Plan de desarrollo 2012-2016. Bogotá: Alcaldía Mayor de Bogotá.

Centro de Investigación para el Desarrollo (CID) (2009). Línea de base para la formulación de la Política de Envejecimiento y Vejez en el Distrito Capital. Bogotá: Universidad Nacional.

Naciones Unidas (2003). Declaración política y Plan de Acción Internacional de Madrid sobre Envejecimiento. Segunda Asamblea Mundial sobre el Envejecimiento. Madrid, España, 8 a 12 de abril de 2002. Nueva York: Naciones Unidas. Recuperado de http://undesadspd.org/Portals/o/ageing/documents/Fulltext-SP.pdf

Secretaría de Integración Social. (2012). Informe de avance sobre la ejecución del plan indicativo de gestión. Bogotá: Alcaldía Mayor de Bogotá.

Secretaría Distrital de Integración Social. (2014). Proyecto 742. Personas mayores. Bogotá: Secretaría Distrital de Integración Social. Recuperado de http://www. integracionsocial.gov.co/index.php?option=com_content\&view=article\&id=8 \&Itemid $=4$

Subdirección Distrital de Integración Social (2009). Manual de inducción y reinducción. Bogotá: Alcaldía Mayor de Bogotá.

Subdirección para la vejez (2012). Proyecto 742. Atencion integral para personas mayores: disminuyendo la discrimnación y segregación social socioeconómica. Bogotá: Alcaldía Mayor de Bogotá. 